\title{
Recurrent sinus of Valsalva aneurysm with thrombogenesis after surgical repair
}

\author{
Meng Zhao ${ }^{1}$, Jieyu Lu ${ }^{2}$, Jingxin $Z h^{1}{ }^{1}$, Yanhu $\mathrm{Wu}^{1}$ \\ ${ }^{1}$ Department of Cardiovascular Surgery, the First Affiliated Hospital of Nanjing Medical University, \\ Nanjing, Jiangsu, China \\ ${ }^{2}$ Nanjing Medical University, Nanjing, Jiangsu, China
}

A 36-year-old woman was admitted to hospital experiencing paroxysmal palpitations for 1 month. Surgical repair of a ruptured sinus of a Valsalva aneurysm was done when she was 10 years old. After admission, the transthoracic echocardiography examination showed that the aortic non-coronary sinus was dilated $(45 \times 36 \mathrm{~mm})$ and a moderately stronger signal could be seen within $(45 \times 17 \mathrm{~mm})$. Coronary computed tomography angiography indicated an expanded aortic non-coronary sinus $(5.3 \times 4.9 \mathrm{~cm})$ with low density areas within (Fig. 1). The coagulation function tests revealed D-dimer $0.8 \mathrm{mg} / \mathrm{L}$. After anesthesia, transesophageal ultrasound identified a mass of thrombus in the expanded sinus. During the operation, the right atrium and aorta were cut open, a massive, grey, smelly purulent thrombus out of the aneurysm was then removed (Suppl. Video 1). The thrombus was sent to the pathology department for pathological tests. Then, a pericardial patch a was used to fix the opening of the aneurysm through the incision of the aorta and another patch $\mathrm{b}$ was used to reinforce it through the rupture in the right atrium. A double helical suture was used to eliminate the sac of the aneurysm. After the operation, transesophageal ultrasound showed that the thrombus had disappeared and that the aneurysm was minimized. Ten days post-surgery, transthoracic echocardiography showed that the expanded non-coronary aneurysm, pre-surgery, was replaced by a hyperechoic mass $(20 \times 21 \mathrm{~mm})$, in which a blood signal could not be

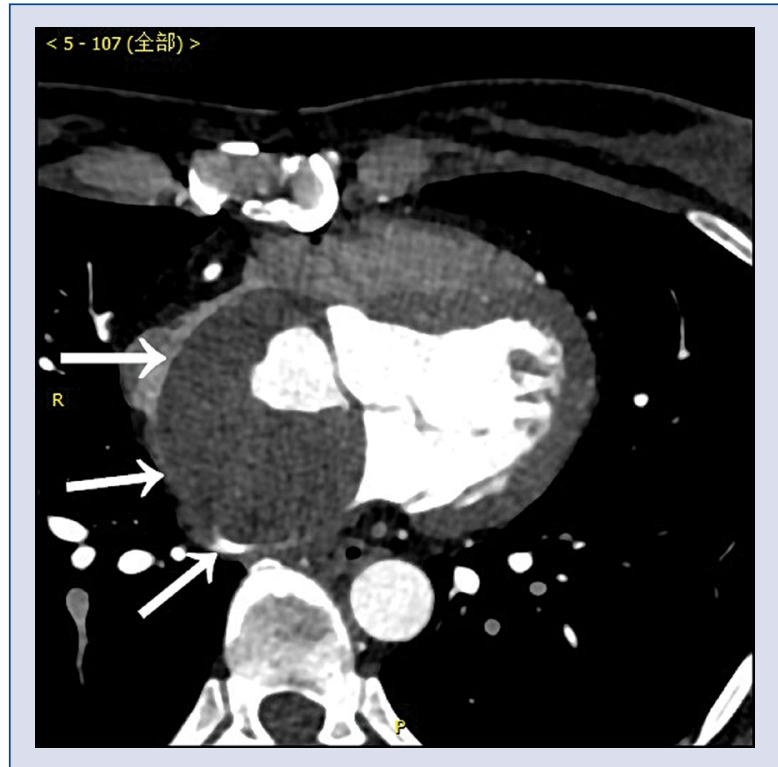

Figure 1. Low density areas can be found in the expanded aortic non-coronary sinus.

detected. Pathological examination of the thrombus indicated fibrous tissue without structure. The patient recovered well and left the hospital successfully.

Funding: This work was financially supported by projects of the National Scientific Foundation of China (NSFC, grant nos.81700340).

Conflict of interest: None declared

Address for correspondence: Yanhu Wu, MD, The First Affiliated Hospital of Nanjing Medical University, Nanjing, Jiangsu Province, China, 210029, tel: +86 13951945999, fax: +86 25 83724440, e-mail: wuyanhu@njmu.edu.cn

Received: $13.04 .2020 \quad$ Accepted: 13.04 .2020 